\title{
Increased putamen volume in adults with autism spectrum disorder
}

\section{Wataru Sato ${ }^{1,2 \dagger}$, Yasutaka Kubota ${ }^{3 * t}$, Takanori Kochiyama ${ }^{1}$, Shota Uono $^{4}$, Sayaka Yoshimura ${ }^{4}$, Reiko Sawada ${ }^{1}$, Morimitsu Sakihama ${ }^{5}$ and Motomi Toichi ${ }^{2,4}$}

${ }^{1}$ The Hakubi Project, Primate Research Institute, Kyoto University, Aichi, Japan

2 The Organization for Promoting Developmental Disorder Research, Kyoto, Japan

${ }^{3}$ Health and Medical Services Center, Shiga University, Shiga, Japan

${ }^{4}$ Faculty of Human Health Science, Graduate School of Medicine, Kyoto University, Kyoto, Japan

${ }^{5}$ Rakuwa-kai Otowa Hospital, Kyoto, Japan

\section{Edited by:}

Bogdan Draganski, University of

Lausanne, Switzerland

\section{Reviewed by:}

Gunther Helms, Lund University, Sweden

Christian Lambert, St. George's

University of London, UK

*Correspondence:

Yasutaka Kubota, Health and Medical Services Center, Shiga University,

1-1-1, Baba, Hikone, Shiga 522-8522,

Japan

e-mail: yka@edu.shiga-u.ac.jp

${ }^{+}$Wataru Sato and Yasutaka Kubota have contributed equally to this work.
Basal ganglia (BG) abnormalities are implicated in the pathophysiology of autism spectrum disorder (ASD). However, studies measuring the volume of the entire BG in individuals with ASD have reported discrepant findings, and no study conducted volume measurement of the entire substructures of the BG (the caudate, putamen, nucleus accumbens, and globus pallidus) in individuals with ASD. We delineated the BG substructures and measured their volumes in 29 adults with ASD without intellectual disabilities and 29 ageand gender-matched typically developed adult controls. We acquired T1-weighted anatomical images and performed semiautomated delineation and volume measurements of the above-mentioned subregions. Total cerebral volumes, sex, and ages were partialed out. Compared with controls, the putamen was significantly larger in the ASD group. The increased volume of the putamen found in high-functioning adults with ASD suggests that structural or histological abnormalities of the putamen may underlie the pathologies of ASD, such as repetitive and stereotyped behaviors and impaired social interactions.

Keywords: autism spectrum disorder (ASD), MRI volumetry, basal ganglia, caudate, putamen, nucleus accumbens and globus pallidus

\section{INTRODUCTION}

Autism spectrum disorders (ASDs) are heterogeneous neurodevelopmental disorders of unknown etiology. The Diagnostic and Statistical Manual-Fourth Edition-Text Revision (DSM-IV-TR) (American Psychiatric Association, 2000) defines autism or autistic disorder as abnormal behavior in the spheres of communication, social relatedness, and stereotyped behaviors within the first 3 years of life (American Psychiatric Association, 2000). The broader category, pervasive developmental disorder (PDD), includes Asperger's disorder and a residual category, pervasive developmental disorder not otherwise specified (PDD-NOS). Asperger's disorder involves pervasive deficits in social interaction and behaviors in the presence of normal verbal development. PDD-NOS is based on social relatedness symptoms but allows for a different age of onset and addresses fewer other spheres. These subcategories are subsumed under the single diagnostic category ASD in the DSM-5 (American Psychiatric Association, 2013).

Repetitive and restrictive behaviors and interests are one of the core clinical manifestations of ASD. It has been postulated that repetitive behaviors/restricted interests are due to abnormalities in the basal ganglia (BG) (Sears et al., 1999; Hikosaka et al., 2002; Hardan et al., 2003; Hollander et al., 2005). The BG is a complex of multiple subcortical regions, including the striatum (composed of three subnuclei: the caudate, putamen, and nucleus accumbens) and the globus pallidus as basic components (Martin, 2003). Abnormalities in the BG have been reported in other neurodevelopmental disorders showing stereotypical motor symptoms, such as obsessive-compulsive disorder (OCD) (Rauch et al., 1997; Kwon et al., 2003; Menzies et al., 2008) and Tourette syndrome (Peterson et al., 2003; Roessner et al., 2011).

The delineation and volume measurement of brain structures using magnetic resonance imaging (MRI) provides valuable information about macroscopic anatomical problems. Previous MRI studies assessing BG volumes in ASD have reported inconsistent findings; some reported no differences in total BG volume (Sears et al., 1999; McAlonan et al., 2002; Hardan et al., 2003), whereas others reported increased volume after controlling for total cerebral volume (TCV) (Herbert et al., 2003; Hollander et al., 2005; Langen et al., 2007). The inconsistent findings suggest that BG abnormalities in ASD are subtle and not apparent by assessing the gross $\mathrm{BG}$ volume. Therefore, more detailed analysis of the BG subregions may be needed. To date, no study conducted anatomical delineation and volume measurement of all the aforementioned subregions of the BG. Previous studies have measured the volumes of some of the BG subregions and identified anatomical abnormalities in the specific BG subregions, although inconsistent, possibly depending on the age group or diagnostic subgroups investigated (Hardan et al., 2003; Hollander et al., 2005, Langen et al., 2007, 2009; Qiu et al., 2010). For example, Langen et al. (2007) measured the caudate, putamen, and nucleus accumbens in individuals with ASD and typically developed controls and found the enlarged volumes in the caudate and putamen in the ASD 
group. A recent study using voxel-based morphometry, which can provide information on microscopic anatomical problems relative to anatomical delineation (Mechelli et al., 2005), also reported that the putamen was larger in adults with ASD (Ecker et al., 2012). Together, these findings suggest that any anatomical abnormality of the BG in adults with ASD might be identified in a specific subregion, such as the putamen.

To investigate this issue, we performed anatomical delineation and volume measurement of the entire BG substructures (the caudate, putamen, nucleus accumbens, and globus pallidus) in adult subjects with ASD without such contaminating factors as intellectual disabilities or language delay, using a semiautomated subcortical segmentation tool for efficient modeling of the brain shape and appearance (Patenaude et al., 2011).

\section{MATERIALS AND METHODS SUBJECTS}

The ASD group comprised 29 adults [5 females, 24 males; age $26.6 \pm 8.2$ (range 18-46) years] without intellectual disabilities, including 12 (2 females, 10 males) with Asperger's disorder and 17 (3 females, 14 males) with PDD-NOS who showed milder symptoms than Asperger's disorder. Therefore, the ASD subjects in this study are considered to have only the core deficits of ASD (i.e., social impairments and repetitive traits). Neurological abnormalities and psychiatric problems other than those associated with ASD (such as clumsiness and circumstantial obsessiveness) were ruled out. The subjects were not taking medication, except one subject on $10 \mathrm{mg}$ paroxetine per day and one on $400 \mathrm{mg}$ sodium valproate per day.

The diagnosis was made using the DSM-IV-TR (American Psychiatric Association, 2000) following a stringent procedure in which every item of the diagnostic criteria was investigated in interviews with the subjects and their parents (and professionals who helped them, if any), conducted by psychiatrists with expertise in developmental disorders (Sayaka Yoshimura, Morimitsu Sakihama, and Motomi Toichi). Only subjects who had at least one of the four social impairment traits (i.e., impairment in nonverbal communication including lack of joint attention, sharing interest, relationships with peers, and emotional and interpersonal mutuality) without satisfying any items of the criteria of autistic disorder, such as language delay, were included. Comprehensive interviews were administered in order to obtain information about the subjects' developmental histories for diagnostic purposes. Of the total 29 individuals with ASD, 25 individuals completed assessments of the intelligence quotients (IQs) using the Wechsler Adult Intelligence Scale-Revised (WAIS-R). Their levels of symptom severity were also assessed by the psychiatrists (Sayaka Yoshimura, Morimitsu Sakihama, and Motomi Toichi) using the Japanese version of Childhood Autism Rating Scale (CARS) (Schopler et al., 1986), the CARS-Tokyo Version (CARS-TV) with satisfactory reliability and validity (Kurita et al., 1989; Tachimori et al., 2003). Handedness was assessed by the Edinburgh Handedness Inventory (Oldfield, 1971).

The control group comprised 29 adults [ 5 females, 24 males; age $25.6 \pm 4.8$ (range $21-43$ ) years]. They were recruited through advertisements and were matched with the ASD group for age and sex. Brief clinical interview with the psychiatrists (Yasutaka
Kubota, Sayaka Yoshimura, and Motomi Toichi) ruled out past or present history of neurological, developmental, or psychiatric problems. The IQs and handedness were assessed as in the ASD group.

All subjects had normal or corrected-to-normal visual acuity. After the procedure and purpose of the study were explained fully, all subjects provided informed consent before participating in the study. This study was approved by the local institutional ethics committee.

\section{MRI ACQUISITION}

Image scanning was performed on a 3-T scanning system at the ATR Brain Activity Imaging Center (Magnetom Trio, A Tim System, Siemens) using a 12-channel array coil without acceleration mode. A T1-weighted high-resolution anatomical image was obtained using a magnetization-prepared rapid gradientecho sequence (TR $2250 \mathrm{~ms}$; TE $3.06 \mathrm{~ms}$; FA 9 $9^{\circ}$; inversion time $1000 \mathrm{~ms}$; FOV $256 \times 256 \mathrm{~mm}$; matrix size $256 \times 256$; voxel size $1 \mathrm{~mm} \times 1 \mathrm{~mm} \times 1 \mathrm{~mm}$; and generalized autocalibrating partially parallel acquisition with an acceleration factor of 2). Elastic pads were placed on each side of the subject's head to stabilize the head position during image acquisition.

\section{IMAGE ANALYSIS}

Segmentation and volumetric analysis of the brain structures was performed with the Integrated Registration and Segmentation Tool (FIRST) in the FMRIB Software Library (FSL) ver. 5 (Patenaude, 2007). FIRST is a semiautomated subcortical segmentation tool used for efficient modeling of the brain shape and appearance within a Bayesian framework (Patenaude et al., 2011). The method has been validated (Patenaude et al., 2011) and has been used in a wide range of applications for volumetric analyses (e.g., Pardini et al., 2014). The analyses were conducted using the standard FIRST procedures (Patenaude et al., 2011). First, the images were registered to a common space based on the Montreal Neurological Institute-152 template using affine registration of the entire head and a subcortical mask to exclude voxels outside the subcortical regions. Then, the inversion transformation was applied to bring the images back into the original space. Finally, the brain structures were segmented based on a Bayesian shape and appearance model, which is part of FSL package and was originally constructed from manually segmented images provided by the Center for Morphometric Analysis, Boston, MA, USA. One of the researchers (Takanori Kochiyama) validated the success of the automated segmentation procedure and inspected the quality of segmentation visually using FSL-provided script to generate summary images in a well-organized webpage format (first_roi_slicesdir). The volumes of the caudate nucleus, putamen, nucleus accumbens, and globus pallidus were extracted for each subject (Figure 1). To control for the TCV, warped modulated gray and white matter images (cf., Ridgway et al., 2011) was obtained using VBM8 software (http://dbm.neuro.uni-jena.de/vbm/) and the sum of gray and white matter volumes was calculated by integrating voxel intensities over the whole segmented image and then multiplying by voxel size. The volumes of each structure were analyzed using a repeated-measures general linear model with group (ASD or control), hemisphere (left or right), sex (male or female), age, and 
TCV as factors. Main effects or interactions related to the group factor were regarded as effects of interest. Further analyses were conducted using a general linear model (1) without the hemisphere factor and (2) with the group factor including three groups (Asperger's disorder, PDD-NOS, or controls). Partial correlations holding the TCV effect constant were also calculated between the mean volumes across both hemispheres and between the volumes and CARS scores [calculating the total scores and composite scores for social communication, social interaction, stereotypes and sensory abnormalities, and emotional regulation proposed by Magyar and Pandolfi (2007)]. We conducted exploratory analyses for the shapes of BG substructures (cf., Patenaude et al., 2011) and the volumes of brain regions other than the BG substructures (Patenaude et al., 2011) using the same software. The results were considered statistically significant at $p<0.05$.

\section{RESULTS}

Background demographics and psychiatric information of the ASD and control groups is summarized in Table 1.

The CARS scores in the ASD group were comparable to those with high-functioning ASD, including Asperger's disorder and PDD-NOS, in previous studies ( $t$-test, $p>0.1$ ) (Koyama et al.,

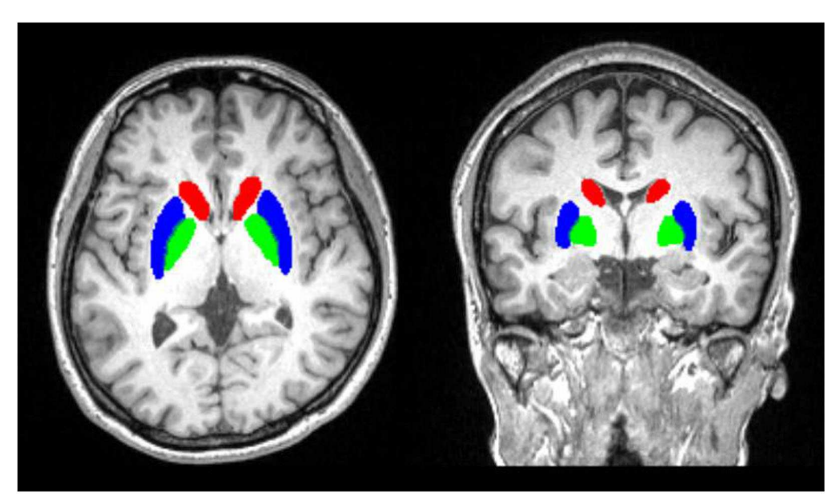

FIGURE 1 | Illustrations of semiautomated subcortical segmentation. The area is overlayed on the anatomical MRI of one of the subjects involved in this study.

Table 1 | Mean (with SD) background information of subjects.

\begin{tabular}{|c|c|c|c|c|c|c|}
\hline & \multicolumn{4}{|l|}{ ASD } & \multirow{2}{*}{\multicolumn{2}{|c|}{ CON }} \\
\hline & Total & & Asperger & NOS & & \\
\hline$n$ total & 29 & & 12 & 17 & 29 & \\
\hline Female & 5 & & 2 & 3 & 5 & \\
\hline Right handed & 29 & & 12 & 17 & 29 & \\
\hline Age & 26.6 & $(8.2)$ & $26.8 \quad(8.8)$ & $26.9 \quad(7.9)$ & 25.6 & $(4.8)$ \\
\hline WAIS-R Full ${ }^{a}$ & 112.6 & (13.9) & $113.0 \quad$ (13.9) & 111.8 (14.9) & 121.5 & $(9.0)$ \\
\hline Verbal & 114.9 & $(16.0)$ & 117.9 (15.3) & 112.5 & 122.3 & $(10.6)$ \\
\hline Performance & 107.4 & (13.2) & $104.3 \quad(11.7)$ & 109.0 & 115.6 & (11.5) \\
\hline CARS $^{a}$ & 22.8 & $(3.0)$ & $24.0 \quad(3.7)$ & $22.0 \quad(2.3)$ & & \\
\hline
\end{tabular}

anvestigations were conducted for 25 subjects in the ASD group.
2007; Uono et al., 2011), indicating that the symptoms in the ASD group were sufficiently severe. There was no significant difference in the CARS scores between ASD subgroups ( $t$-test, $p>0.1$ ). The full-scale IQs of both groups fell within the normal range. All subjects were right handed.

For the putamen volumes (Figure 2), a general linear model analysis with group, hemisphere, sex, age, and TCV as factors revealed a significant main effect of group $[F(1,52)=4.61$, $p<0.05]$, which indicated a higher volume for the ASD group than for the control group. There was no other significant main effect or interaction $(p>0.1)$. To confirm the group difference in the putamen volumes in each hemisphere, we repeated the analysis without the hemisphere factor for the data for each hemisphere, and found that the main effects of group were significant in both hemispheres $[F(1,52)=4.10, p<0.05]$. For the volumes of the caudate nucleus, nucleus accumbens, and globus pallidus (Figure 2), there were no significant main effects or interactions $(p>0.1)$.

To test the effect of ASD subgroup (Figure S1 in Supplementary Material), a further general linear model was evaluated for the putamen volumes using the same design, except that the group factor included three levels (Asperger's disorder, PDD-NOS, and controls) and contrasts were conducted across groups. The results confirmed a marginally significant difference between Asperger's disorder and the controls $(p<0.1)$ and a significant difference between PDD-NOS and the controls $(p<0.05)$, but none between Asperger's disorder and PDD-NOS $(p>0.1)$.

To test the influence of IQ on putamen volume, partial correlations holding the TCV effect constant were calculated between the mean putamen volumes across both hemispheres and the fullscale/verbal/performance IQs in the ASD group. There were no significant partial correlations between the putamen volumes and IQs (partial $r s=-0.14,-0.03$, and -0.26 for the full-scale, verbal, and performance IQs, respectively; all $p>0.1$ ).

To test the relationships between the putamen volumes and psychiatric symptoms, partial correlations (controlling for the effect of TCV) were calculated between the mean putamen volumes across both hemispheres and CARS scores in the ASD group (Table $\mathrm{S} 1$ in Supplementary Material). There were no significant partial correlations between the putamen volumes and CARS total score (partial $r=-0.22, p>0.1$ ) or any of Magyar and Pandolfi's (2007) composite scores (absolute partial $r<0.33, p>0.1$ ).

We conducted some additional exploratory analyses. First, since the software allows analysis of the form of the brain regions, we analyzed the forms of the BG substructures (Figure S2 in Supplementary Material). There were no significant differences between the ASD and control groups in the forms of the caudate nucleus, putamen, nucleus accumbens, and globus pallidus. Then, because the software can output the volumes of other brain structures, including the thalamus, hippocampus, and amygdala of each hemisphere (Table S2 in Supplementary Material), we analyzed the volumes of these regions using the same methods. There were no significant main effects or interactions related to the group factor for any regions (all $p>0.1$ ).

\section{DISCUSSION}

To our knowledge, this is the first delineation study on the whole basic BG substructures (the caudate nucleus, putamen, nucleus 

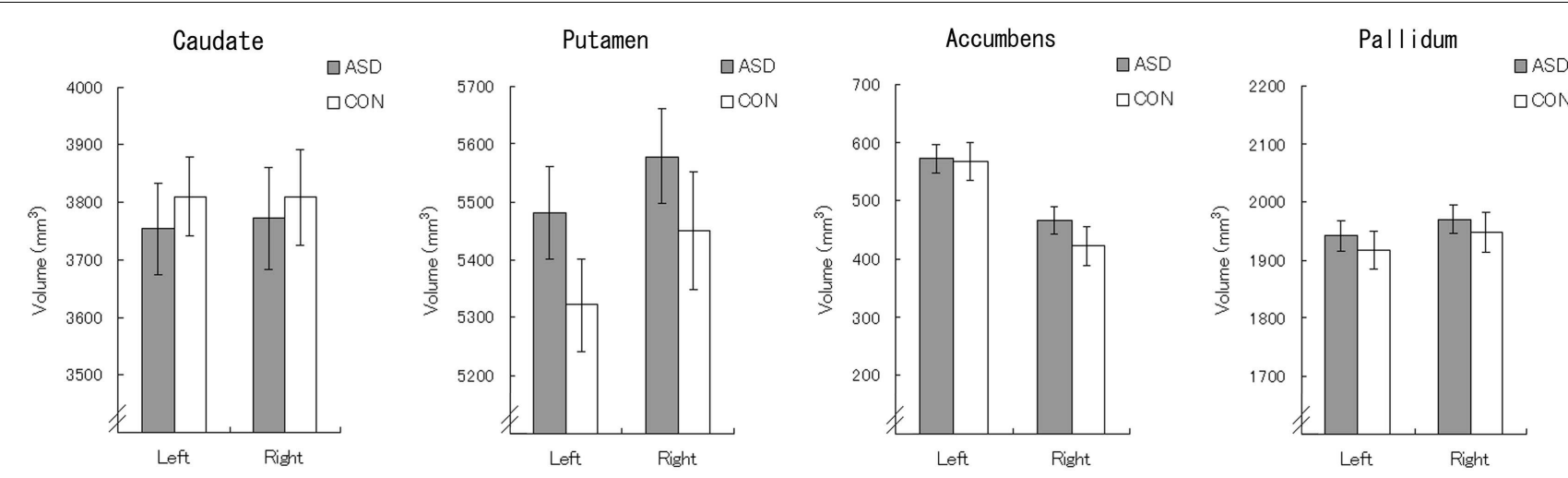

FIGURE 2 | Mean ( \pm SEM) volumes of the caudate, putamen, globus pallidus, and nucleus accumbens. ${ }^{*} p<0.05$

accumbens, and globus pallidus) that identified increased putamen volume in well-characterized adults with ASD. Individuals with ASD have problems with social interaction and non-verbal communication as children and adults. Although follow-up studies report improved adaptive functioning in adulthood (Magiati et al., 2014), many adults with ASD have considerable psychosocial impairment (Hofvander et al., 2009; Mordre et al., 2012). Our findings are important for elucidating the neuroanatomical basis of the residual autistic traits in adults in this population.

The present results of increased putamen volumes in ASD are consistent with the findings in several previous anatomical delineation studies (e.g., Langen et al., 2007) and voxel-based morphometry studies (e.g., Ecker et al., 2012). However, other anatomical delineation studies (e.g., Hollander et al., 2005; Qiu et al., 2010) and voxel-based morphometry studies (e.g., Nickl-Jockschat et al., 2012; Greimel et al., 2013) reported different results. These inconsistent findings may be partly attributable to some methodological differences, such as differences in ages, diagnostic subcategories, and data analyses. Specifically, Qiu et al. (2010) tested 32 boys aged 8-12 with ASD and reported a non-significant trend toward a putamen volume decrease. These discrepancies may be attributable to the difference in the developmental stage. Typically developing male children show an increase in the putamen with age, even after adjusting for TCV (Giedd et al., 1996). A recent longitudinal study observed that the growth rate of the striatum was faster in 9- to 12-year-old children with ASD compared to typically developing children, and faster striatal growth was correlated with more severe repetitive behavior at preschool ages (Langen et al., 2014). Since the ASD subjects of the current study were adults, accelerated growth of the putamen volumes may be more pronounced than those of previous studies. Hollander et al. (2005) reported volume increases in the caudate and a near-threshold trend of the putamen volume increases in 17 adults with ASD. These results may be accounted for by differences in subgroups. While Hollander et al. (2005) included nine with autistic disorder, seven with Asperger's disorder, and one with PDD-NOS, our subjects included 12 subjects with Asperger's disorder and 17 subjects with PDD-NOS. The caudate volume increases were reported mostly in studies on individuals with autistic disorders [Sears et al., 1999; Rojas et al., 2006; Langen et al., 2014; for review, see
Stanfield et al. (2008)]. These findings might reflect more distinct autistic traits in these populations, such as motor stereotypes as well as abnormalities in verbal/communicative domain. Greimel et al. (2013) conducted whole-brain voxel-based morphometry analysis on 26 subjects with Asperger syndrome, 20 with highfunctioning autism, and one with atypical autism according to ICD-10 (World Health Organization, 1993), but did find enlarged volumes in the putamen compared to controls. The discrepancy may be explained from the fact that the whole-brain voxel-based morphometry approach is less sensitive in finding differences in an a priori specified region in the ROI-based approach. Given anatomical abnormalities in ASD distributed in a structure such as the putamen, there may be no single voxel in a general linear model analysis that survives statistical threshold. Further studies using other suitable methodology for analyzing subcortical structures, such as machine learning-based automated segmentation (Powell et al., 2008) are warranted.

Our correlation data using CARS measures did not show clear evidence about the functional significance of this anatomical problem in the ASD group. The results could be accounted for the limitation that CARS lack the items for a repetitive and stereotyped pattern of behaviors (Schopler et al., 1986). Regarding this issue, a previous study reported that total putamen volumes were positively correlated with repetitive behavior scores in ASD (Hollander et al., 2005). Anatomical abnormalities of the putamen were also reported in a variety of psychiatric/neurodevelopmental disorders showing repetitive/obsessive symptoms, including OCD, Tourette syndrome, and schizophrenic disorders (Chemerinski et al., 2013). For example, increased putamen volumes were repeatedly observed in subjects with OCD [for review, see Rotge et al. (2010)]. Together with these findings, we speculate that BG pathology confined to the putamen may be related to the repetitive behavior/restricted interest in our subjects, but as yet it is not possible to define the direction of this association. Further studies are needed in order to investigate possible relationship between the observed abnormality in the putamen and the clinical phenotype of repetitiveness in adult individuals with ASD.

The human putamen receives associative, motor, and limbic projections (Haber, 2003; Bernácer et al., 2012) and performs integrative information processing underlying various tasks, such as 
motor control (Lehéricy et al., 2006) and cognitive and emotional processing (Monchi et al., 2001; Haber et al., 2006). Interestingly, functional neuroimaging studies of ASD demonstrate abnormal activity of the striate (the lateral parts correspond to the putamen and caudate) during social processing (Dapretto et al., 2006; Masten et al., 2011; Weng et al., 2011; Delmonte et al., 2012) and reward processing tasks (Scott-Van Zeeland et al., 2010; Delmonte et al., 2012). Furthermore, diffusion tensor imaging studies have shown decreased white matter connectivity between the striatum and prefrontal cortex in ASD compared with typically developed individuals (Langen et al., 2012). These data suggest that the putamen abnormality observed in ASD might be related not only to the domain of motor/repetitive behaviors per se but also to broader ASD phenotypes, such as impairments in higher cognitive functioning, including emotional facial recognition and reciprocal social interaction.

Several limitations of this study need to be mentioned. First, it included a modest number of high-functioning adults with Asperger's disorder or PDD-NOS, and the results should be interpreted with caution. Since our sample did not include subjects with autistic disorder, it is not clear whether our findings can be extended to low-functioning adults with autistic disorder. Second, the use of a cross-sectional design limits the developmental conclusions that can be drawn from the present findings. Further longitudinal studies are needed to explore the specific neurodevelopmental pattern of putamen growth in ASD. Finally, the volumetric approach is clearly limited in clarifying the functional abnormality of the BG that is involved in broader cortico-subcortical networks. Further neuroimaging and electrophysiological studies targeting possible relationship between neural circuitries involving the putamen and higher cognitive processing in ASD, especially in the domain of social interaction and/or decision making, are needed.

\section{ACKNOWLEDGMENTS}

The authors thank Emi Yokoyama, Akemi Inoue, and Kazusa Minemoto for help with subject recruitment. This study was supported by funds from the Japan Society for the Promotion of Science (JSPS) Program for Next Generation WorldLeading Researchers No. LZ008, the Sakamoto Research Institute of Psychopathology, and the Organization for Promoting Developmental Disorder Research.

\section{SUPPLEMENTARY MATERIAL}

The Supplementary Material for this article can be found online at http://www.frontiersin.org/Journal/10.3389/fnhum.2014.00957/ abstract

\section{REFERENCES}

American Psychiatric Association. (2000). Diagnostic and Statistical Manual of Mental Disorders: DSM-IV-TR ${ }^{\circledR}, 4$ th Edn. Washington, DC: American Psychiatric Association.

American Psychiatric Association. (2013). Diagnostic and Statistical Manual of Mental Disorders: DSM-5, 5th Edn. Arlington, VA: American Psychiatric Association.

Bernácer, J., Prensa, L., and Giménez-Amaya, J. M. (2012). Distribution of GABAergic interneurons and dopaminergic cells in the functional territories of the human striatum. PLoS ONE 7:e30504. doi:10.1371/journal.pone.0030504
Chemerinski, E., Byne, W., Kolaitis, J. C., Glanton, C. F., Canfield, E. L., Newmark, R. E., et al. (2013). Larger putamen size in antipsychotic-naïve individuals with schizotypal personality disorder. Schizophr. Res. 143, 158-164. doi:10.1016/j.schres.2012.11.003

Dapretto, M., Davies, M. S., Pfeifer, J. H., Scott, A. A., Sigman, M., Bookheimer, S. Y., et al. (2006). Understanding emotions in others: mirror neuron dysfunction in children with autism spectrum disorders. Nat. Neurosci. 9, 28-30. doi:10.1038/nn1611

Delmonte, S., Balsters, J. H., McGrath, J., Fitzgerald, J., Brennan, S., Fagan, A. J., et al. (2012). Social and monetary reward processing in autism spectrum disorders. Mol. Autism 3, 7. doi:10.1186/2040-2392-3-7

Ecker, C., Suckling, J., Deoni, S. C., Lombardo, M. V., Bullmore, E. T., Baron-Cohen, S., et al. (2012). Brain anatomy and its relationship to behavior in adults with autism spectrum disorder: a multicenter magnetic resonance imaging study. Arch. Gen. Psychiatry 69, 195-209. doi:10.1001/archgenpsychiatry.2011.1251

Giedd, J. N., Snell, J. W., Lange, N., Rajapakse, J. C., Casey, B. J., Kozuch, P. L., et al. (1996). Quantitative magnetic resonance imaging of human brain development: ages 4-18. Cereb. Cortex 6, 551-560. doi:10.1093/cercor/6.4.551

Greimel, E., Nehrkorn, B., Schulte-Rüther, M., Fink, G. R., Nickl-Jockschat, T., Herpertz-Dahlmann, B., et al. (2013). Changes in grey matter development in autism spectrum disorder. Brain Struct. Funct. 218, 929-942. doi:10.1007/ s00429-012-0439-9

Haber, S. N. (2003). The primate basal ganglia: parallel and integrative networks. J. Chem. Neuroanat. 26, 317-330. doi:10.1016/j.jchemneu.2003.10.003

Haber, S. N., Kim, K.-S., Mailly, P., and Calzavara, R. (2006). Reward-related cortical inputs define a large striatal region in primates that interface with associative cortical connections, providing a substrate for incentive-based learning. J. Neurosci. 26, 8368-8376. doi:10.1523/JNEUROSCI.0271-06.2006

Hardan, A. Y., Kilpatrick, M., Keshavan, M. S., and Minshew, N. J. (2003). Motor performance and anatomic magnetic resonance imaging (MRI) of the basal ganglia in autism. J. Child Neurol. 18, 317-324. doi:10.1177/08830738030180050801

Herbert, M. R., Ziegler, D. A., Deutsch, C. K., O’Brien, L. M., Lange, N., Bakardjiev, A., et al. (2003). Dissociations of cerebral cortex, subcortical and cerebral white matter volumes in autistic boys. Brain 126(Pt 5), 1182-1192. doi:10.1093/brain/awg110

Hikosaka, O., Nakamura, K., Sakai, K., and Nakahara, H. (2002). Central mechanisms of motor skill learning. Curr. Opin. Neurobiol. 12, 217-222. doi:10.1016/ S0959-4388(02)00307-0

Hofvander, B., Delorme, R., Chaste, P., Nydén, A., Wentz, E., Ståhlberg, O., et al. (2009). Psychiatric and psychosocial problems in adults with normal-intelligence autism spectrum disorders. BMC Psychiatry 9, 35. doi:10.1186/1471-244X-9-35

Hollander, E., Anagnostou, E., Chaplin, W., Esposito, K., Haznedar, M. M., Licalzi, E., et al. (2005). Striatal volume on magnetic resonance imaging and repetitive behaviors in autism. Biol. Psychiatry. 58, 226-232. doi:10.1016/j.biopsych.2005. 03.040

Koyama, T., Tachimori, H., Osada, H., Takeda, T., and Kurita, H. (2007). Cognitive and symptom profiles in Asperger's syndrome and high-functioning autism. Psychiatry Clin. Neurosci. 61, 99-104. doi:10.1111/j.1440-1819.2007.01617.x

Kurita, H., Miyake, Y., and Katsuno, K. (1989). Reliability and validity of the Childhood Autism Rating Scale-Tokyo Version (CARS-TV). J. Autism Dev. Disord. 19, 389-396. doi:10.1007/BF02212937

Kwon, J. S., Kim, J.-J., Lee, D. W., Lee, J. S., Lee, D. S., Kim, M.-S., et al. (2003). Neural correlates of clinical symptoms and cognitive dysfunctions in obsessivecompulsive disorder. Psychiatry Res. 122, 37-47. doi:10.1016/S0925-4927(02) 00104-X

Langen, M., Bos, D., Noordermeer, S. D. S., Nederveen, H., van Engeland, H., and Durston, S. (2014). Changes in the development of striatum are involved in repetitive behavior in autism. Biol. Psychiatry 76, 405-11. doi:10.1016/j.biopsych. 2013.08.013

Langen, M., Durston, S., Staal, W. G., Palmen, S. J. M. C., and Van Engeland, H. (2007). Caudate nucleus is enlarged in high-functioning medication-naive subjects with autism. Biol. Psychiatry 62, 262-266. doi:10.1016/j.biopsych.2006.09. 040

Langen, M., Leemans, A., Johnston, P., Ecker, C., Daly, E., Murphy, C. M., et al. (2012). Fronto-striatal circuitry and inhibitory control in autism: findings from diffusion tensor imaging tractography. Cortex 48, 183-193. doi:10.1016/j.cortex. 2011.05.018 
Langen, M., Schnack, H. G., Nederveen, H., Bos, D., Lahuis, B. E., de Jonge, M. V., et al. (2009). Changes in the developmental trajectories of striatum in autism. Biol. Psychiatry 66, 327-333. doi:10.1016/j.biopsych.2009.03.017

Lehéricy, S., Bardinet, E., Tremblay, L., Van de Moortele, P.-F., Pochon, J.-B., Dormont, D., et al. (2006). Motor control in basal ganglia circuits using fMRI and brain atlas approaches. Cereb. Cortex 16, 149-161. doi:10.1093/cercor/ bhi089

Magiati, I., Tay, X. W., and Howlin, P. (2014). Cognitive, language, social and behavioural outcomes in adults with autism spectrum disorders: a systematic review of longitudinal follow-up studies in adulthood. Clin. Psychol. Rev. 34, 73-86. doi:10.1016/j.cpr.2013.11.002

Magyar, C. I., and Pandolfi, V. (2007). Factor structure evaluation of the childhood autism rating scale. J. Autism Dev. Disord. 37, 1787-1794. doi:10.1007/s10803006-0313-9

Martin, J. H. (2003). Neuroanatomy: Text and Atlas. New York: McGraw-Hill Medical.

Masten, C. L., Colich, N. L., Rudie, J. D., Bookheimer, S. Y., Eisenberger, N. I., and Dapretto, M. (2011). An fMRI investigation of responses to peer rejection in adolescents with autism spectrum disorders. Dev. Cogn. Neurosci. 1, 260-270. doi:10.1016/j.dcn.2011.01.004

McAlonan, G. M., Daly, E., Kumari, V., Critchley, H. D., van Amelsvoort, T., Suckling, J., et al. (2002). Brain anatomy and sensorimotor gating in Asperger's syndrome. Brain 125(Pt 7), 1594-1606. doi:10.1093/brain/awf150

Mechelli, A., Price, C. J., Friston, K. J., and Ashburner, J. (2005). Voxel-based morphometry of the human brain: methods and applications. Curr. Med. Imag. Rev. 1, 105-113. doi:10.2174/1573405054038726

Menzies, L., Chamberlain, S. R., Laird, A. R., Thelen, S. M., Sahakian, B. J., and Bullmore, E. T. (2008). Integrating evidence from neuroimaging and neuropsychological studies of obsessive-compulsive disorder: the orbitofronto-striatal model revisited. Neurosci. Biobehav. Rev. 32, 525-549. doi:10.1016/j.neubiorev. 2007.09.005

Monchi, O., Petrides, M., Petre, V., Worsley, K., and Dagher, A. (2001). Wisconsin card sorting revisited: distinct neural circuits participating in different stages of the task identified by event-related functional magnetic resonance imaging. $J$. Neurosci. 21, 7733-7741.

Mordre, M., Groholt, B., Knudsen, A. K., Sponheim, E., Mykletun, A., and Myhre, A. M. (2012). Is long-term prognosis for pervasive developmental disorder not otherwise specified different from prognosis for autistic disorder? Findings from a 30-year follow-up study. J. Autism Dev. Disord. 42, 920-928. doi:10.1007/s10803-011-1319-5

Nickl-Jockschat, T., Habel, U., Michel, T. M., Manning, J., Laird, A. R., Fox, P. T., et al. (2012). Brain structure anomalies in autism spectrum disorder - a meta-analysis of VBM studies using anatomic likelihood estimation. Hum. Brain Mapp. 33, 1470-1489. doi:10.1002/hbm.21299

Oldfield, R. C. (1971). The assessment and analysis of handedness: the Edinburgh Inventory. Neuropsychologia 9, 97-113. doi:10.1016/0028-3932(71)90067-4

Pardini, D. A., Raine, A., Erickson, K., and Loeber, R. (2014). Lower amygdala volume in men is associated with childhood aggression, early psychopathic traits, and future violence. Biol. Psychiatry 75, 73-80. doi:10.1016/j.biopsych. 2013.04.003

Patenaude, B. (2007). Bayesian Statistical Models of Shape and Appearance for Subcortical Brain Segmentation. University of Oxford. Retrieved from: http: //ethos.bl.uk/OrderDetails.do?uin=uk.bl.ethos.491674

Patenaude, B., Smith, S. M., Kennedy, D. N., and Jenkinson, M. (2011). A Bayesian model of shape and appearance for subcortical brain segmentation. Neuroimage 56, 907-922. doi:10.1016/j.neuroimage.2011.02.046

Peterson, B. S., Thomas, P., Kane, M. J., Scahill, L., Zhang, H., Bronen, R., et al. (2003). Basal ganglia volumes in patients with Gilles de la Tourette syndrome. Arch. Gen. Psychiatry 60, 415-424. doi:10.1001/archpsyc.60.4.415

Powell, S., Magnotta, V. A., Johnson, H., Jammalamadaka, V. K., Andreasen, N. C., and Pierson, R. (2008). Registration and machine learning-based automated segmentation of subcortical and cerebellar brain structures. Neuroimage 39, 238-247. doi:10.1016/j.neuroimage.2007.05.063

Qiu, A., Adler, M., Crocetti, D., Miller, M. I., and Mostofsky, S. H. (2010). Basal ganglia shapes predict social, communication, and motor dysfunctions in boys with autism spectrum disorder. J. Am. Acad. Child Adolesc. Psychiatry 49, 539-551, 551.e1-551.e4. doi:10.1016/j.jaac.2010.02.012

Rauch, S. L., Savage, C. R., Alpert, N. M., Dougherty, D., Kendrick, A., Curran, T., et al. (1997). Probing striatal function in obsessive-compulsive disorder: a PET study of implicit sequence learning. J. Neuropsychiatry Clin. Neurosci. 9, 568-573.

Ridgway, G., Barnes, J., Pepple, T., and Fox, N. (2011). Estimation of total intracranial volume; a comparison of methods. Alzheimer's Dementia. 7, S62-S63. doi:10.1016/j.jalz.2011.05.099

Roessner, V., Overlack, S., Schmidt-Samoa, C., Baudewig, J., Dechent, P., Rothenberger, A., et al. (2011). Increased putamen and callosal motor subregion in treatment-naïve boys with Tourette syndrome indicates changes in the bihemispheric motor network. J. Child Psychol. Psychiatry 52, 306-314. doi:10.1111/j. 1469-7610.2010.02324.x

Rojas, D. C., Peterson, E., Winterrowd, E., Reite, M. L., Rogers, S. J., and Tregellas, J. R. (2006). Regional gray matter volumetric changes in autism associated with social and repetitive behavior symptoms. BMC Psychiatry 6:56. doi:10.1186/1471-244X-6-56

Rotge, J.-Y., Langbour, N., Guehl, D., Bioulac, B., Jaafari, N., Allard, M., et al. (2010). Gray matter alterations in obsessive-compulsive disorder: an anatomic likelihood estimation meta-analysis. Neuropsychopharmacology 35, 686-691. doi:10.1038/npp.2009.175

Schopler, E., Reichler, R. J., and Renner, B. R. (1986). The Childhood Autism Rating Scale (CARS): For Diagnostic Screening and Classification of Autism. New York: Irvington.

Scott-Van Zeeland, A. A., Dapretto, M., Ghahremani, D. G., Poldrack, R. A., and Bookheimer, S. Y. (2010). Reward processing in autism. Autism Res. 3, 53-67. doi:10.1002/aur.122

Sears, L. L., Vest, C., Mohamed, S., Bailey, J., Ranson, B. J., and Piven, J. (1999). An MRI study of the basal ganglia in autism. Prog. Neuropsychopharmacol. Biol. Psychiatry 23, 613-624. doi:10.1016/S0278-5846(99)00020-2

Stanfield, A. C., McIntosh, A. M., Spencer, M. D., Philip, R., Gaur, S., and Lawrie, S. M. (2008). Towards a neuroanatomy of autism: a systematic review and metaanalysis of structural magnetic resonance imaging studies. Eur. Psychiatry 23, 289-299. doi:10.1016/j.eurpsy.2007.05.006

Tachimori, H., Osada, H., and Kurita, H. (2003). Childhood autism rating scaleTokyo version for screening pervasive developmental disorders. Psychiatry Clin. Neurosci. 57, 113-118. doi:10.1046/j.1440-1819.2003.01087.x

Uono, S., Sato, W., and Toichi, M. (2011). The specific impairment of fearful expression recognition and its atypical development in pervasive developmental disorder. Soc. Neurosci. 6, 452-463. doi:10.1080/17470919.2011.605593

Weng, S. J., Carrasco, M., Swartz, J. R., Wiggins, J. L., Kurapati, N., Liberzon, I., et al. (2011). Neural activation to emotional faces in adolescents with autism spectrum disorders. J. Child Psychol. Psychiatry 52, 296-305. doi:10.1111/j.14697610.2010.02317.x

World Health Organization. (1993). The ICD-10 Classification of Mental and Behavioural Disorders: Diagnostic Criteria for Research. Geneva: World Health Organization.

Conflict of Interest Statement: The authors declare that the research was conducted in the absence of any commercial or financial relationships that could be construed as a potential conflict of interest.

Received: 01 May 2014; accepted: 10 November 2014; published online: 25 November 2014.

Citation: Sato W, Kubota Y, Kochiyama T, Uono S, Yoshimura S, Sawada R, Sakihama $M$ and Toichi $M$ (2014) Increased putamen volume in adults with autism spectrum disorder. Front. Hum. Neurosci. 8:957. doi: 10.3389/fnhum.2014.00957

This article was submitted to the journal Frontiers in Human Neuroscience. Copyright (c) 2014 Sato, Kubota, Kochiyama, Uono, Yoshimura, Sawada, Sakihama and Toichi. This is an open-access article distributed under the terms of the Creative Commons Attribution License (CC BY). The use, distribution or reproduction in other forums is permitted, provided the original author(s) or licensor are credited and that the original publication in this journal is cited, in accordance with accepted academic practice. No use, distribution or reproduction is permitted which does not comply with these terms. 